\title{
Polysaccharides and virulence of Burkholderia pseudomallei
}

\author{
M. Sarkar-Tyson, ${ }^{1}$ J. E. Thwaite, ${ }^{1}$ S. V. Harding, ${ }^{1}$ S. J. Smither, ${ }^{1}$ \\ P. C. F. Oyston, ${ }^{1}$ T. P. Atkins ${ }^{1}$ and R. W. Titball ${ }^{1,2}$ \\ ${ }^{1}$ Defence Science and Technology Laboratory, Porton Down, Salisbury SP4 OJO, UK \\ ${ }^{2}$ Department of Infectious and Tropical Disease, London School of Hygiene and Tropical Medicine, \\ Keppel Street, London WC1E 7HT, UK
}

Correspondence

M. Sarkar-Tyson

mstyson@dstl.gov.uk

Received 1 November 2006

Accepted 6 March 2007

\begin{abstract}
Burkholderia pseudomallei is the causative agent of melioidosis, an infectious disease of humans and animals. Gene clusters which encode capsular polysaccharide (type I O-PS) and LPS (type II O-PS), both of which play roles in virulence, have previously been identified. Here, the identification of two further putative clusters, type III O-PS and type IV O-PS, is reported. Mice challenged with type III O-PS or type IV O-PS mutants showed increased mean times to death (7.8 and 11.6 days) compared to those challenged with wild-type B. pseudomallei (3 days). To investigate the possible roles of polysaccharides in protection, mice were immunized with killed cells of wild-type B. pseudomallei or killed cells of B. pseudomallei with mutations in the $O$ antigen, capsular polysaccharide, type III O-PS or type IV O-PS gene clusters. Immunization with all polysaccharide mutant strains resulted in delayed time to death compared to the naïve controls, following challenge with wild-type B. pseudomallei strain K96243. However, immunization with killed polysaccharide mutant strains conferred different degrees of protection, demonstrating the immunological importance of the polysaccharide clusters on the surface of $B$. pseudomallei.
\end{abstract}

\section{INTRODUCTION}

Burkholderia pseudomallei is a Gram-negative saprophytic rod which is particularly prevalent in the soil and standing water in Southeast Asia and Northern Australia (Dance, 1990; Currie, 2003). It is the causative agent of melioidosis, an infectious disease of both humans and animals. Melioidosis can present itself in a number of forms including acute septicaemia, acute pulmonary infection, and subacute and chronic diseases (Currie, 2003; White, 2003; Cheng \& Currie, 2005). In some cases, a latent infection is established which may reactivate to cause disease in later life. The factors that influence the presentation of disease are not known, but differences in the virulence of strains might contribute to the outcome of infection.

Currently there is no vaccine to protect against B. pseudomallei and little is known about the virulence mechanisms of the pathogen. However, B. pseudomallei expresses surface polysaccharides, two of which have been well characterized: the capsular polysaccharide and LPS, also known as type I O-PS and type II O-PS, respectively (Deshazer et al., 1998; Reckseidler et al., 2001; Perry et al., 1995). Previous studies have shown that LPS $\mathrm{O}$ antigen and capsular polysaccharide mutants of $B$. pseudomallei are markedly attenuated in animal models of disease (Deshazer et al., 1998; Atkins et al., 2002; Reckseidler et al., 2001). The

Abbreviations: i.p., intraperitoneal; MTTD, mean time to death.
B. pseudomallei $\mathrm{O}$ antigen appears to have properties typical of bacterial $\mathrm{O}$ antigens, for example by providing resistance to serum killing (Deshazer et al., 1998). The function of the capsular polysaccharide is less clear, but it may provide resistance to phagocytosis by reducing levels of complement C3 deposition (Reckseidler-Zenteno et al., 2005). The role of the capsular polysaccharide in virulence is supported by the observation that the encoding gene cluster is present in the related human pathogen Burkholderia mallei (Deshazer et al., 2001). In contrast, the gene cluster is absent in Burkholderia thailandensis, a non-pathogenic environmental saprophyte that is genetically closely related to B. pseudomallei (Ong et al., 2004; Kespichayawattana et al., 2004; Brett et al., 1998; Yu et al., 2006).

The potential immunological importance of these polysaccharides has also been demonstrated by the reduction in the lethality of infection in mice and diabetic rats when passively immunized with antibodies to the LPS or capsular polysaccharide (Bryan et al., 1994; Jones et al., 2002). Furthermore, immunization of mice with LPS or capsular polysaccharide provides protection against a subsequent challenge with $B$. pseudomallei, suggesting that these polysaccharides have potential as vaccine candidates (Nelson et al., 2004). The relative roles of the polysaccharides in $B$. pseudomallei virulence remain unclear, and have been further complicated by the identification of two further putative polysaccharide clusters, encoding type III O-PS 
and type IV O-PS, in the B. pseudomallei strain K96243 genome sequence (Holden et al., 2004). In support of the possibility that $B$. pseudomallei expresses polysaccharides other than LPS and capsular, a study conducted with 1327 strains has shown that $3 \%$ of strains have either another LPS serotype which has a different $\mathrm{O}$-antigen ladder profile, or a rough form of LPS that lacks an O-antigen ladder (Anuntagool et al., 2006). Another exopolysaccharide has been reported (Nimtz et al., 1997); however, the operon encoding this polysaccharide has not been identified.

In this study, the role of each polysaccharide gene cluster in virulence and protection was investigated by insertionally inactivating genes within each operon.

\section{METHODS}

Bacterial strains. B. pseudomallei strain K96243 was grown in LuriaBertani (LB) broth at $37^{\circ} \mathrm{C}$ with shaking for $18 \mathrm{~h}$. For viable counts, media were solidified with $15 \mathrm{~g}$ Bacto agar $\mathrm{l}^{-1}$. Escherichia coli strains DH5 $\alpha$ ppir and E19851 were grown in LB broth, supplemented with $50 \mu \mathrm{g}$ kanamycin $\mathrm{ml}^{-1}$ (Sigma) as required, with shaking for $18 \mathrm{~h}$.

PCR. Primers used to amplify partial sequences from $w b i A, w c b H$, BPSS0421 and BPSS1833 genes of B. pseudomallei are described in Table 1. The partial genes were amplified under the following conditions: denaturation at $95^{\circ} \mathrm{C}$ for $10 \mathrm{~min}$, followed by 30 cycles of denaturation at $95{ }^{\circ} \mathrm{C}$ for $1 \mathrm{~min}$, annealing at $50{ }^{\circ} \mathrm{C}$ for $1 \mathrm{~min}$ and extension for $1 \mathrm{~min}$, followed by final extension at $72{ }^{\circ} \mathrm{C}$ for $10 \mathrm{~min}$. Amplified PCR products were initially cloned into pCR-Blunt IITOPO (Invitrogen) and subsequently cloned into the SmaI or EcoRV site of the pKNOCK vector (Alexeyev, 1999). Restriction sites were engineered onto both the forward and reverse primers for subsequent digestion with either SmaI or EcoRV, creating blunt products for cloning into pKNOCK. The recombinant pKNOCK plasmids were transformed into E. coli strain E19851.

Conjugation of B. pseudomallei strain K96243. Overnight cultures of recombinant E. coli E19851 or B. pseudomallei were centrifuged at $3000 \mathrm{~g}$ for $10 \mathrm{~min}$ at room temperature. Supernatants were discarded, and the pellets were resuspended in half the original volume. A $10 \mu \mathrm{l}$ aliquot of each culture was pipetted onto a sterile nitrocellulose membrane on an LB agar plate and incubated for 6-8 h at $37{ }^{\circ} \mathrm{C}$. The nitrocellulose membrane was vortexed in $1 \mathrm{ml} \mathrm{LB}$ medium, and $100 \mu \mathrm{l}$ aliquots were plated onto LB agar plates supplemented with $100 \mu \mathrm{g}$ ampicillin and $700 \mu \mathrm{g}$ kanamycin. The plates were incubated for 2 days at $37^{\circ} \mathrm{C}$.
Southern blotting. Genomic DNA was prepared from $100 \mathrm{ml}$ cultures of B. pseudomallei in LB broth by a lysozyme, phenol/chloroform extraction method (Mack \& Titball, 1998). B. pseudomallei genomic DNA $(5 \mu \mathrm{g})$ was digested with the selected restriction endonuclease, separated by gel electrophoresis using a $0.8 \%(\mathrm{w} / \mathrm{v})$ agarose gel, and transferred to a positively charged nylon membrane (Hybond- $\mathrm{N}^{+}$; Amersham Biosciences). Southern hybridization was performed using the Roche DIG labelling Southern kit following the manufacturer's instructions.

Animals. Female BALB/c age-matched mice, approximately 6 weeks old, were used in this study. The mice were grouped together in cages of five with free access to food and water, and subjected to a $12 \mathrm{~h}$ light/dark cycle. For challenge with viable B. pseudomallei, the animals were handled under biosafety level III containment conditions within a half-suit isolator, compliant with British Standard BS5726. All investigations involving animals were carried out according to the requirements of the Animal (Scientific Procedures) Act 1986. Humane end points were strictly observed, and animals deemed incapable of survival were humanely killed by cervical dislocation.

Virulence studies. Groups of five mice were challenged with $1 \times 10^{4}$ c.f.u. of wild-type B. pseudomallei strain K96243, B. pseudomallei K96243 $\triangle B P S S 0421:$ :kan (type III O-PS) or B. pseudomallei K96243 $\triangle B P S S 1833:$ :kan (type IV O-PS) by the intraperitoneal (i.p.) route, and the infection was monitored for 5 weeks.

Determination of bacterial hydrophobicity. The hydrophobicity of bacterial strains was measured using the bacterial adhesion to hydrocarbon (BAH) test (Beck et al., 1988; Rosenberg, 1984). All experiments were carried out using overnight bacterial cell suspensions, washed twice in sodium phosphate buffer $(\mathrm{pH}$ 6.8), and resuspended to $\mathrm{OD}_{440}$ 1.0. Bacterial suspensions $(3 \mathrm{ml})$, prepared in sodium phosphate buffer ( $\mathrm{pH} 6.8$ ), were overlaid with $1 \mathrm{ml}$ hexadecane (Sigma). After $1 \mathrm{~min}$ of vigorous agitation, the phases were allowed to separate for $15 \mathrm{~min}$ at room temperature. Partitioning in the hydrocarbon phase was calculated as follows:

$\left\{\left[\mathrm{OD}_{440}\right.\right.$ (original bacterial suspension) $-\mathrm{OD}_{440}$ (aqueous phase)]/ $\mathrm{OD}_{440}$ (original bacterial suspension) $\} \times 100$

From each bacterial strain, the percentage hydrophobicity was obtained from the mean values of three separate experiments; the error bars on Fig. 3 represent the mean \pm SD.

Heat-inactivation of bacteria. B. pseudomallei strains were grown in $\mathrm{LB}$ broth at $37{ }^{\circ} \mathrm{C}$ overnight with agitation. The cultures were adjusted with PBS to the same $\mathrm{OD}_{590}$ value and harvested by centrifugation at $5000 \mathrm{~g}$ for $15 \mathrm{~min}$. Bacteria were washed once in the original culture volume of PBS, harvested again, and then

Table 1. Primers used in this study to construct mutant strains

\begin{tabular}{|c|c|}
\hline Strain & Primer \\
\hline 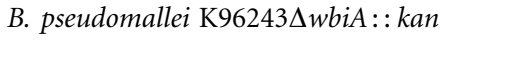 & $\begin{array}{l}\text { wbiA.KO.For - ggactagtggatgcgttgcagtttgcgt } \\
\text { wbiA.KO.Rev - gaagcttgatgccaactgaaccatgcg }\end{array}$ \\
\hline 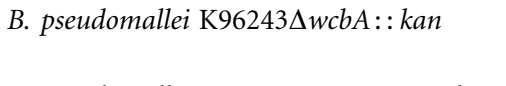 & $\begin{array}{l}w c b H . K O . F o r ~-~ g g g g a t c c c g a t a t g g a c a t g g a g c c t g \\
w c b H . K O . R e v ~-~ g g a a g c t t c c a a c t t c t c c g c a t a g t c g\end{array}$ \\
\hline B. pseudomallei K96243 $\triangle B P S S 0421$ :: kan & $\begin{array}{l}\text { BPSS0421.KO.For - gatatcggaactgcagcacatggtgg } \\
\text { BPSS0421.KO.Rev - gatatcggtagaaactcagcgtcgcg }\end{array}$ \\
\hline 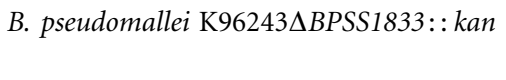 & $\begin{array}{l}\text { BPSS1833.KO.For - cccgggcgatcgggtagtactcgatg } \\
\text { BPSS1833.KO.Rev - cccgggcgagttcacgaagtatgcgg }\end{array}$ \\
\hline
\end{tabular}


resuspended in the original volume of PBS. Bacteria were heatinactivated by incubating in a water bath maintained at $80{ }^{\circ} \mathrm{C}$ for $3 \mathrm{~h}$. Inactivated bacteria were then placed at $4{ }^{\circ} \mathrm{C}$ for at least $16 \mathrm{~h}$. Prior to heat-inactivation, a viable count was performed to determine the number of heat-killed bacteria in c.f.u. $\mathrm{ml}^{-1}$. Inactivation was confirmed by culturing $10 \%$ of each inactivated culture in LB broth for 7 days at $37^{\circ} \mathrm{C}$, then plating out the broth on LB agar plates for 7 days at $37{ }^{\circ} \mathrm{C}$ to confirm that no viable bacteria remained.

Immunization and challenge. Groups of $10 \mathrm{BALB} / \mathrm{c}$ mice were immunized by the i.p. route with $10^{8}$ c.f.u. bacteria on day 0 , followed by boosts administered on days 14 and 28. A period of 5 weeks elapsed prior to i.p. challenge with $6.05 \times 10^{4}$ c.f.u. wild-type bacteria, and the animals were monitored for a further 5 weeks.

\section{RESULTS AND DISCUSSION}

\section{Organization of polysaccharide gene clusters in B. pseudomallei strain K96243}

Previously, gene clusters encoding the capsular polysaccharide (type I O-PS) and O antigen (type II O-PS) have been identified, and mutations in these gene clusters shown to attenuate B. pseudomallei (Atkins et al., 2002; Deshazer et al., 1998; Reckseidler et al., 2001). Two additional polysaccharide clusters of unknown function have also been identified in the genome sequence of $B$. pseudomallei strain K96243 (Holden et al., 2004). To correspond with the existing nomenclature of the polysaccharides in $B$. pseudomallei, these novel clusters would encode type III O-PS and type IV O-PS. The putative type III O-PS polysaccharide would be encoded by a cluster of 13 genes located between BPSS0417 and BPSS0429. The genes have been assigned putative functions related to polysaccharide biosynthesis and transport (Holden et al., 2004). Genes BPSS0419-BPSS421 show homology to the $r f b$ cluster of Salmonella typhimurium and the asc gene cluster of Yersinia pseudotuberculosis, both of which are involved in $\mathrm{O}$-antigen biosynthesis (Brahmbhatt et al., 1988; Thorson et al., 1994). The gene cluster which encodes polysaccharide O-PS IV consists of eight genes from BPSS1825 to BPSS1832 with putative functions of capsular polysaccharide biosynthesis and polysaccharide transport proteins. The type III O-PS and type IV O-PS clusters are not present in B. mallei, but part of the clusters is conserved in B. thailandensis, indicating that these putative polysaccharide clusters have a possible role in environmental survival.

The possibility that B. pseudomallei expresses polysaccharides other than type I O-PS and type II O-PS has been raised by several previous studies (Kawahara et al., 1998; Masoud et al., 1997; Nimtz et al., 1997; Steinmetz et al., 1995, 2000). For example, one polysaccharide distinct from type I O-PS and type II O-PS has been reported to be a linear tetrasaccharide repeating unit consisting of three galactose residues, one bearing a 2-linked $\mathrm{O}$-acetyl group and a 3-deoxy-D-manno-2-2-octulosonic acid residue
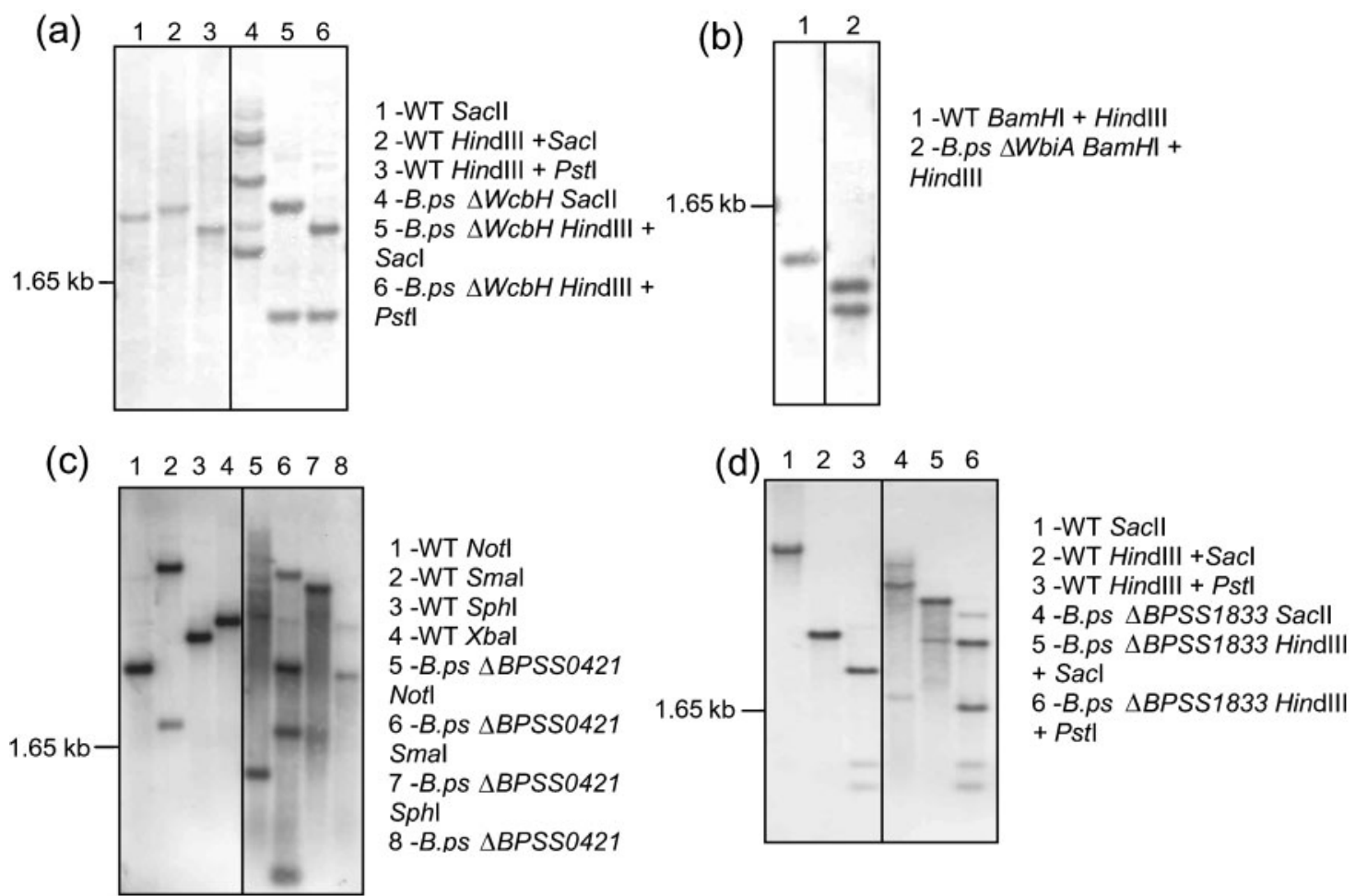

Fig. 1. Southern blotting confirms the disruption of the polysaccharide clusters in the B. pseudomallei mutant strains. (a) Capsule operon, wbiA gene; (b) LPS operon, wcbH gene; (c) PS-III, BPSS0421; and (d) PS-IV, BPSS1833. 


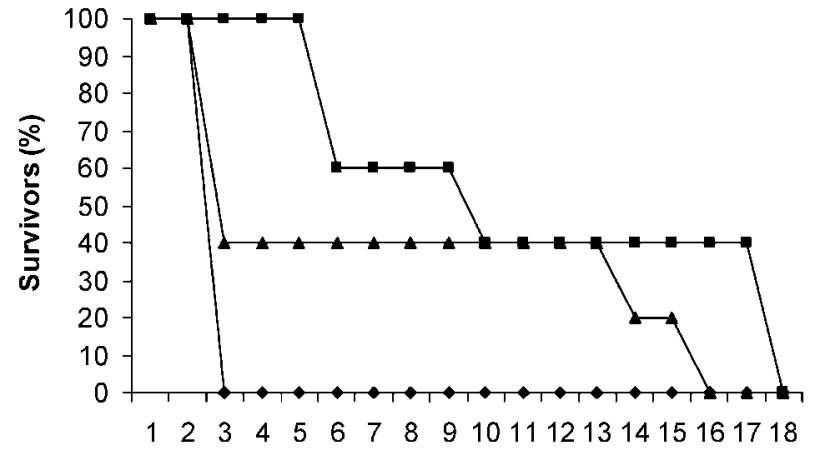

Days Post Infection

Fig. 2. Survival of mice infected with $10^{4}$ c.f.u. wild-type $(\boldsymbol{)})$, B. pseudomallei $\triangle B P S S 0421$ (A) and B. pseudomallei $\triangle B P S S 1833(\mathbf{\square})$.

(Masoud et al., 1997; Nimtz et al., 1997). Screening of this polysaccharide using a monoclonal antibody has shown expression in all B. pseudomallei strains tested, and also in B. mallei (Steinmetz et al., 1995). The absence of the putative polysaccharide clusters type III O-PS and type IV $\mathrm{O}-\mathrm{PS}$ in B. mallei indicates that these clusters do not encode this polysaccharide.

\section{Construction of mutants}

B. pseudomallei mutants were constructed by insertionally inactivating a gene located in each of the polysaccharide gene clusters. Homologous regions from the trans-acylase gene wbiA, the mannosyltransferase gene $w c b H, B P S S 0421$ and the BPSS1833 gene were cloned into the suicide vector pKNOCK and transformed into E. coli (Alexeyev, 1999). The suicide plasmid constructs were introduced into wildtype B. pseudomallei strain K96243 by conjugation, creating

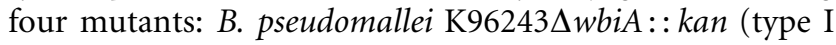
O-PS), B. pseudomallei K96243 $\Delta w c b H::$ kan (type II OPS), B. pseudomallei K96243 $\triangle$ BPSS0421::kan (type III O-PS) and B. pseudomallei K96243 $\triangle B P S S 1833:$ : kan (type IV O-PS). Correct integration of the plasmid into each polysaccharide biosynthesis operon was confirmed by Southern blotting (Fig. 1).

\section{Disruption of the type IV O-PS but not the type III O-PS gene cluster significantly attenuates $B$. pseudomallei}

The attenuation of type I O-PS or type II O-PS mutants of $B$. pseudomallei in mice is well documented (Atkins et al., 2002; Deshazer et al., 1998; Reckseidler et al., 2001). To investigate the roles of the putative type III O-PS or type IV O-PS polysaccharides in virulence, mice were challenged by the i.p. route with $10^{4}$ c.f.u. $B$.

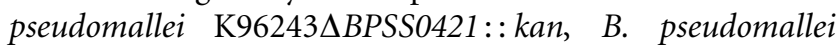
K96243 $B$ BPSS1833: : kan or with wild-type B. pseudomallei (Fig. 2). Animals in the group which were challenged with wild-type bacteria died with a mean time to death (MTTD) of 3 days. There was a delayed time to death in the groups challenged with the mutant strain $B$. pseudomallei K96243 $B P S S 1833:$ : kan with a MTTD of 11.6 days. The difference in survival between wild-type and $B$. pseudomallei K96243 $\triangle B P S S 1833:$ : kan was statistically significant using the Mantel-Haenszel log rank test $(P=0.0027)$, indicating that this polysaccharide cluster plays a role in the virulence of $B$. pseudomallei. Although we could not demonstrate a role in virulence for $B$. pseudomallei K96243 $B P S S 0421:: k a n$ in this experiment, the group challenged with this mutant strain showed survival for up to 15 days compared to the group challenged with wildtype bacteria, in which death occurred within 3 days.

The type III O-PS and type IV O-PS genes are absent in B. mallei but partially present in B. thailandensis, which may reflect the different lifestyles of these three related species. The B. pseudomallei K96243 genome comprises two chromosomes of 4.07 and $3.17 \mathrm{Mb}$ in size (Holden et al., 2004). The gene clusters encoding the LPS and capsular polysaccharides are present on chromosome 1 . The genes on chromosome 1 are generally associated with cell growth and metabolism. Chromosome 2 is thought to encode functions associated with adaptation; both of the gene clusters which encode the putative type III O-PS and type IV O-PS are present on chromosome 2.

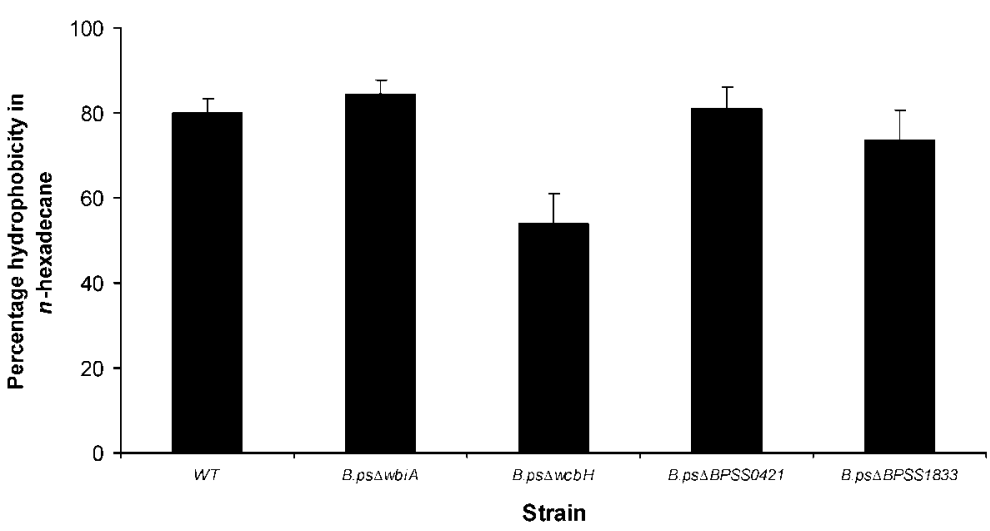

Fig. 3. Surface hydrophobicity of $B$. pseudomallei strain K96243 wild-type (WT) and the polysaccharide mutant strains. An ANOVA table with Bonferroni's multiple comparison test showed that the difference between $B$. pseudomallei wild-type and the capsule mutant strain was significant $(P<0.001)$. 
Table 2. Survival of BALB/c mice, vaccinated with heat-killed strains and subsequently challenged with $6.05 \times 10^{4}$ c.f.u. wild-type B. pseudomallei

\begin{tabular}{|c|c|c|c|}
\hline Vaccination & $\begin{array}{c}\text { Polysaccharide cluster } \\
\text { disrupted }\end{array}$ & $\begin{array}{l}\text { Survivors } \\
(\%)\end{array}$ & $\begin{array}{r}\text { MTTD } \\
\text { (days) }\end{array}$ \\
\hline B. pseudomallei K96243 & None & 40 & 26.6 \\
\hline 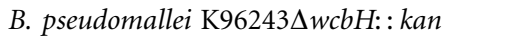 & Type I O-PS (capsule) & 70 & 32.5 \\
\hline B. pseudomallei K962434wbiA: : kan & Type II O-PS (LPS) & 80 & 33 \\
\hline B. pseudomallei K96243 $\triangle$ BPSS0421: : kan & Type III O-PS & 50 & 29.7 \\
\hline B. pseudomallei K96243 $B$ BSS1833: : kan & Type IV O-PS & 50 & 28.3 \\
\hline PBS control & & 10 & 9.2 \\
\hline
\end{tabular}

\section{Only the capsular polysaccharide affects the hydrophobic surface of $B$. pseudomallei}

The ability of the polysaccharides to modify the surface hydrophobicity of $B$. pseudomallei was investigated using the BAH method, based on the degree to which bacterial cells can partition between water (aqueous phase) and hexadecane (organic phase) (Fig. 3). Only the capsule

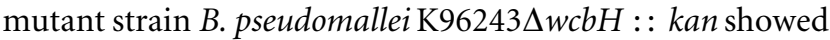
a different hydrophobicity to that of the wild-type strain. There are reports of a link between the surface hydrophobicity of Gram-negative and Gram-positive bacteria and the potential to cause disease (Vercauteren et al., 1993; Ofek, 1994). It is thought that a hydrophobic cell surface may be an advantage to pathogenic bacteria in vivo by promoting interactions with host cells. B. thailandensis does not produce capsular polysaccharide, and it has been reported that the ability of $B$. pseudomallei to adhere to and invade human epithelial cells is significantly higher than that of B. thailandensis (Ong et al., 2004; Kespichayawattana et al., 2004; Brett et al., 1998).

\section{Immunization with the LPS- or capsule-negative strains results in the highest levels of protection against wild-type $B$. pseudomallei}

The relative level of protection contributed by each polysaccharide was evaluated using heat-killed whole cells. Heat-inactivated wild-type or mutant cells were used to immunize groups of 10 mice three times at 2-weekly intervals, followed by i.p. challenge with $10 \times$ MLD of wild-type B. pseudomallei K96243, and the animals were monitored for 35 days (Table 2). Nine of 10 control mice challenged with wild-type bacteria died, and the MTTD of these animals was 9.2 days. One mouse survived to day 35 but demonstrated signs of disease. The MTTD of mice in the immunized groups was increased approximately threefold. There was $40 \%$ survival in the group vaccinated with wild-type $B$. pseudomallei, and groups immunized with the mutant strains B. pseudomallei K96243 $B P S S 0421$ :: kan and B. pseudomallei K96243 $B P S S 1833:$ : kan also showed similar survival of $50 \%$ in each group. Interestingly, groups vaccinated with the mutant strains $B$. pseudomallei K96243 4 wbiA:: kan and B. pseudomallei K96243$\Delta w c b H:$ :kan had higher levels of survival with 80 and
$70 \%$, respectively, of mice still remaining alive at day 35. All the survivors in the vaccinated groups demonstrated no signs of disease. The observed increase in protection levels could be due to the exposure of other protective antigens on the bacterial cell surface, or increased expression of the remaining polysaccharides.

We aimed to investigate the roles of these polysaccharides in protection using heat-killed cells. Heat-killed cells have previously been shown to offer protection against a $B$. pseudomallei challenge by the i.p. route (Razak, 1986). The differences in the levels of protection afforded by each group may be an indication of the relative importance of each polysaccharide in protection. Surprisingly, although the capsule and LPS have previously been shown to be protective antigens, immunization with heat-killed cells with mutations in the type I O-PS or type II O-PS gene clusters provides enhanced protection compared to that with heat-killed wild-type cells (Nelson et al., 2004). This could reflect the exposure of other protective surface antigens due to removal of the highly abundant polysaccharide, or it may be due to upregulation of one or more of the remaining polysaccharides. This finding merits additional investigation.

\section{REFERENCES}

Alexeyev, M. F. (1999). The pKNOCK series of broad-host-range mobilizable suicide vectors for gene knockout and targeted DNA insertion into the chromosome of Gram-negative bacteria. Biotechniques 26, 824-826.

Anuntagool, N., Wuthiekanun, V., White, N. J., Currie, B. J., Sermswan, R. W., Wongratanacheewin, S., Taweechaisupapong, S., Chaiyaroj, S. C. \& Strisinha, S. (2006). Lipopolysaccharide heterogeneity among Burkholderia pseudomallei from different geographic and clinical origins. Am J Trop Med Hyg 74, 348-352.

Atkins, T. P., Prior, R. G., Mack, K., Russell, P., Nelson, M., Prior, J., Ellis, J., Oyston, P. C., Dougan, G. \& Titball, R. W. (2002). Characterisation of an acapsular mutant of Burkholderia pseudomallei identified by signature tagged mutagenesis. J Med Microbiol 51, 539-547.

Beck, G., Puchelle, E., Plotkowski, C. \& Peslin, R. (1988). Effect of growth on surface-charge and hydrophobicity of Staphylococcus aureus. Ann Inst Pasteur Microbiol 139, 655-664.

Brahmbhatt, H. N., Wyk, P., Quigley, N. B. \& Reeves, P. R. (1988). Complete physical map of the $\mathrm{Rfb}$ gene-cluster encoding 
biosynthetic-enzymes for the O-antigen of Salmonella typhimurium Lt2. J Bacteriol 170, 98-102.

Brett, P. J., Deshazer, D. \& Woods, D. (1998). Burkholderia thailandensis sp. nov., a Burkholderia pseudomallei-like species. Int J Syst Bacteriol 48, 317-320.

Bryan, L. E., Wong, S. E., Woods, D., Dance, D. \& Chaowagul, W. (1994). Passive protection of diabetic rats with antisera specific for the polysaccharide portion of the lipopolysaccharide isolated from Pseudomonas pseudomallei. Can J Infect Dis 5, 170-178.

Cheng, A. C. \& Currie, B. J. (2005). Melioidosis: epidemiology, pathophysiology and management. Clin Microbiol Rev 18, 383-416.

Currie, B. J. (2003). Melioidosis: an important cause of pneumonia in residents of and travellers returned from endemic regions. Eur Respir J 22, 542-550.

Dance, D. A. (1990). Melioidosis. Rev Med Microbiol 1, 143-150.

Deshazer, D., Brett, P. \& Woods, D. (1998). The type II O-antigenic polysaccharide moiety of Burkholderia pseudomallei lipopolysaccharide is required for serum resistance and virulence. Mol Microbiol 30, 1081-1100.

Deshazer, D., Wagg, D., Fritz, D. \& Woods, D. (2001). Identification of a Burkholderia mallei polysaccharide gene cluster by subtractive hybridisation and demonstration that the encoded capsule is an essential virulence determinant. Microb Pathog 30, 253-269.

Holden, M. T. G., Titball, R. W., Peacock, S. J., Cerdeno-Tarraga, A. M., Atkins, T., Crossman, L. C., Pitt, T., Churcher, C., Mungall, K. \& other authors (2004). Genomic plasticity of the causative agent of melioidosis, Burkholderia pseudomallei. Proc Natl Acad Sci U S A 101, 14240-14245.

Jones, S. M., Ellis, J. F., Russell, P., Griffin, K. F. \& Oyston, P. C. F. (2002). Passive protection against Burkholderia pseudomallei infection in mice by monoclonal antibodies against capsular polysaccharide, lipopolysaccharide or proteins. J Med Microbiol 51, 1055-1062.

Kawahara, K., Dejsirilert, S. \& Ezaki, T. (1998). Characterisation of three capsular polysaccharides produced by Burkholderia pseudomallei. FEMS Microbiol Lett 169, 283-287.

Kespichayawattana, W., Intachote, P., Utaisincharoen, P. \& Sirisinha, S. (2004). Virulent Burkholderia pseudomallei is more efficient than avirulent Burkholderia thailandensis in invasion of and adherence to cultured human epithelial cells. Microb Pathog 36, 287-292.

Mack, K. \& Titball, R. (1998). The detection of insertion sequences within the human pathogen Burkholderia pseudomallei which have been identified previously in Burkholderia cepacia. FEMS Microbiol Lett 162, 69-74.

Masoud, H., Ho, M., Schollaardt, T. \& Perry, M. (1997). Characterization of the capsular polysaccharide of Burkholderia (Pseudomonas) pseudomallei 304b. J Bacteriol 179, 5663-5669.

Nelson, M., Prior, J. L., Lever, M. S., Jones, H. E., Atkins, T. P. \& Titball, R. W. (2004). Evaluation of lipopolysaccharide and capsular polysaccharide as subunit vaccines against experimental melioidosis. J Med Microbiol 53, 1177-1182.

Nimtz, M., Wray, V., Domke, T., Brenneke, B., Haussler, S. \& Steinmezt, I. (1997). Structure of an acidic exopolysaccharide of Burkholderia pseudomallei. Eur J Biochem 250, 608-616.

Ofek, I. \& Doyle, R. J. (1994). Bacterial Adhesion to Cells and Tissues. New York: Chapman \& Hall.

Ong, C., Ooi, C. H., Wang, D. L., Chong, H. L., Ng, K. C., Rodrigues, F., Lee, M. A. \& Tan, P. (2004). Patterns of large-scale genomic variation in virulent and avirulent Burkholderia species. Genome Res 14, 2295-2307.

Perry, M. B., MacLean, L. L., Schollaardt, T., Bryan, L. E. \& Ho, M. (1995). Structural characterisation of the lipopolysaccharide O antigens of Burkholderia pseudomallei. Infect Immun 63, 3348-3352.

Razak, C. E., Ismail, G., Embi, N. \& Omar, O. (1986). Protection studies using whole cells and partially purified toxic material (PPTM) of Pseudomonas pseudomallei. Malays Appl Biol 15, 105-111.

Reckseidler, S L., Deshazer, D., Sokol, P. \& Woods, D. (2001). Detection of bacterial virulence genes by subtractive hybridisation: identification of capsular polysaccharide of Burkholderia pseudomallei as a major virulence determinant. Infect Immun 69, 34-44.

Reckseidler-Zenteno, S. L., DeVinney, R. \& Woods, D. E. (2005). The capsular polysaccharide of Burkholderia pseudomallei contributes to survival in serum by reducing complement factor C3b deposition. Infect Immun 73, 1106-1115.

Rosenberg, M. (1984). Bacterial adherence to hydrocarbons - a useful technique for studying cell-surface hydrophobicity. FEMS Microbiol Lett 22, 289-295.

Steinmetz, I., Rohde, M. \& Brenneke, B. (1995). Purification and characterization of an exopolysaccharide of Burkholderia (Pseudomonas) pseudomallei. Infect Immun 63, 3959-3965.

Steinmetz, I., Nimtz, M., Wray, V., Haubler, S., Reganzerowski, A. \& Brenneke, B. (2000). Exopolysaccharides of Burkholderia pseudomallei. Acta Trop 74, 211-214.

Thorson, J. S., Lo, S. F., Ploux, O., He, X. M. \& Liu, H. W. (1994). Studies of the biosynthesis of 3,6-dideoxyhexoses - molecular-cloning and characterization of the Asc (Ascarylose) region from YersiniaPseudotuberculosis serogroup Va. J Bacteriol 176, 5483-5493.

Vercauteren, R., Dom, P. \& Haesebrouck, F. (1993). Virulence of bacteria in relation to their hydrophobicity, adhesiveness and phagocytosis. Vlaams Diergeneeskd Tijdschr 62, 29-34.

White, N. J. (2003). Melioidosis. Lancet 361, 1715-1722.

Yu, Y., Kim, H. S., Chua, H. H., Linm, C. H., Sim, S. H., Lin, D., Derr, A., Engels, R., DeShazer, D. \& other authors (2006). Genomic patterns of pathogen evolution revealed by comparison of Burkholderia pseudomallei, the causative agent of melioidosis, to avirulent Burkholderia thailandensis. BMC Microbiol 6, 46. 